\title{
Competencias interculturales en Educación Superior: Aspecto clave para la movilidad
}

\section{Intercultural Competences in Higher Education: A Key for Mobility}

Mar Adalid Donat, Carmen Carmona Rodríguez, José Vidal Mollón,

María Jesús Benlloch Sanchis

\{marado; mabensan\}@alumni.uv.es, (https://orcid.org/0000-0002-9276-0984), (https://orcid.org/ 0000-0001-8227-9591)

\{carmen.carmona; jose.vidal-mollon\}@uv.es (https://orcid.org/0000-0003-1183-7255), (https://orcid.org/0000-0001-8308-5748)

Facultad de Filosofía y Ciencias de la Educación. Departamento de Métodos de Investigación y Diagnóstico en Educación. Universitat de València, España. (+34)963864330

\section{Resumen}

El objetivo principal de este trabajo es analizar las diferencias en competencias interculturales entre el alumnado de la Universitat de València que desea solicitar el programa Erasmus y alumnado que no desea solicitarlo. Mediante un cuestionario se realizó la recogida de información de estudiantes procedentes del área de ciencias sociales de la Universitat de València. En general, los resultados muestran que existen diferencias entre estos dos grupos de estudiantes. El alumnado que va a solicitar el programa de movilidad Erasmus tiene mayor competencia comunicativa en diferentes idiomas, y mayor apertura mental en comparación con el alumnado que no tiene interés en solicitar este tipo de becas. Aunque los resultados indican diferencias en estas dos competencias, es necesario resaltar el papel de las competencias interculturales y comunicativas para el desarrollo integral de las personas y la socialización e integración en los nuevos contextos de diversidad cultural.

\section{Palabras Clave}

Educación superior; Movilidad; Competencias interculturales; Estudiantes

\begin{abstract}
The main objective of this study is to analyze the differences in intercultural competencies among the students at the university who wish to apply for the Erasmus program and those who do not. A questionnaire was used to gather information from students who study Social Sciences at the University of Valencia. In general, results show differences between these two groups. Students with an interest in applying for the exchange program of Erasmus had a greater communicative competence in different languages and were more open minded than students lacking an interest in applying for this kind of scholarship. Although results show only differences in the former two competences, we need to highlight that intercultural and communication competences are fundamental in personal development, socialization and integration in new and diverse cultural contexts.
\end{abstract}

\section{Keywords}

Higher Education; Mobility; Intercultural Competencies; Students 


\section{Introducción}

La situación actual de crisis en España ha llevado a que uno de los principales motivos para emigrar sea el actual desempleo. Los datos indican que existe un gran porcentaje de desempleo que se sitúa en el $18.63 \%$, y en particular de desempleo juvenil un 42.92\%, (INE, 2017).

Este hecho ha supuesto que cientos de miles de jóvenes, y no tan jóvenes, hayan tenido que emigrar a países de dentro y fuera de la Unión Europea. Aproximadamente la población española residente en el extranjero desde el 2009 hasta el 2016 es de un total de 2.305.030 personas (INE, 2017). Estas personas han de enfrentarse a distintos desafíos como: un nuevo idioma, cultura, estructura social y laboral. En concreto, según la fuente citada, los países a los que se emigra principalmente son Francia, Reino Unido, Alemania, y Estados Unidos.

Por tanto, emigrar, más que una posibilidad, es la única opción para una gran mayoría de los jóvenes, a los cuales también necesitamos formar en competencias que les faciliten el proceso adaptativo al nuevo destino y cultura.

La cultura ha sido definida por filósofos, antropólogos y sociólogos, pero una de las definiciones más significativas es la de Giddens que declara que:

"La cultura tiene que ver con las formas de vida de los miembros de una sociedad o de sus grupos. Incluye el modo de vestir, las costumbres matrimoniales y la vida familiar, las pautas laborales, las ceremonias religiosas y los pasatiempos" (Giddens, 1991, p. 21).

Pero la cultura no es únicamente un conjunto de costumbre y actividades, tiene también diversas funciones. La cultura permite, por un lado, que el individuo tenga una identidad cultural fundamentada en unos valores, creencias y principios que le facilitan su desarrollo intelectual y le permiten establecer relaciones con el resto de personas, ya sean de su misma cultura o de otra. Además, para conocer, respetar y tolerar otras identidades culturales es necesario reconocer primero la propia. Por otro lado, otra de las funciones fundamentales de la cultura es permitir, a través de un dialogo fundamentado en los mismos principios, actitudes y costumbres, solucionar las necesidades y problemas que puedan sucederse en la sociedad (Sáenz, 2015).

Por ello es necesario analizar nuevos contextos multiculturales y convertirlos en contextos interculturales, donde exista el conocimiento y comprensión de los otros, favoreciendo que la cultura pueda continuar ejerciendo las funciones que le pertenecen y que no podrían desempeñarse de no existir una integración de todas las culturas coexistentes (Escámez, 1994).

Pero la cultura no es rígida, y que exista diversidad cultural es enriquecedor y necesario para el progreso, por tanto: 
"La comprensión entre las culturas y entre los miembros de diversas culturas es posible, si se dan determinadas condiciones, porque las culturas no son realidades estáticas sino dinámicas, y los miembros de una cultura, a pesar de la configuración de la personalidad recibida de ella, son entre sí más diferentes y flexibles de lo que ciertos planteamientos etnográficos, de antropología cultural y de psicosociología pretenden demostrar" (Escámez, 1994, p. 30).

\subsection{Choque cultural}

Debido a los recientes, y ya no tan recientes, movimientos de personas a través del mundo estableciendo su destino en un lugar diferente al de origen, se han creado nuevos contextos donde coexisten dos o muchas más culturas. A este tipo de movimientos o flujos de personas se les ha denominado 'migraciones'. Independientemente de la duración de la estancia en el país ajeno, existe un periodo en el cual se es inmigrante. Alves y De la Peña (2013) coinciden en que la persona que emigra desde su país de origen a otro lleva consigo una carga cultural muy fuerte, y cuando se establece en el país de destino se puede producir un fenómeno llamado 'choque cultural'. Este proceso consiste en la sensación de ansiedad que se produce en el individuo como resultado de la pérdida del contacto directo con la cultura de origen.

En el contexto de Educación Superior, por ejemplo, cuando un estudiante cursa un programa educativo en el extranjero, puede también sufrir un choque cultural, dado que se traslada a un contexto nuevo, otra cultura y situaciones que le pueden crear una mayor incertidumbre.

Por tanto, la persona que se traslada estará expuesta a nuevos estímulos culturales desconocidos para ella, que además deberá interpretarlos, comprenderlos y adaptarse a ellos (Alves y De la Peña, 2013). Para que el choque no sea muy fuerte, pese a veces ser inevitable y necesario, existen acciones o pautas para que sea menor, algunas de ellas son: haber estado en contacto con otras culturas previamente, la similitud entre la cultura de origen y la nueva, el conocimiento que se tiene de esta (por ejemplo, si se conoce el idioma, habrá menor probabilidad de malentendidos) o las competencias, actitudes y valores del sujeto, serán determinantes en el nivel de afectación del choque cultural.

Por tanto, el choque cultural es el momento en el que dos culturas entran en contacto, y se analiza la percepción y nivel de ansiedad que se genera sobre el individuo cuando esto sucede. Este fenómeno, es necesario diferenciarlo del proceso de aculturación (Berry, 2005; 2008; Ward y Kennedy, 1994). El primero, hace referencia a la situación que se da cuando el individuo llega a la nueva sociedad receptora, y el segundo se encarga de definir las diferentes formas de coexistir que se pueden dar cuando el individuo está instalado y ha superado el impacto del choque cultural. 


\subsection{Aculturación}

El término aculturación ha sido estudiado desde diferentes perspectivas a lo largo del tiempo y no siempre ha tenido la misma acepción. Inicialmente, se entendía que la aculturación se trataba de un proceso unidireccional en el cual solo la población inmigrante sufría cambios durante el periodo de adaptación, hasta alcanzar ciertos niveles de contacto y valores culturales correspondientes a la sociedad donde se habría realizado la inmersión y hasta considerarse estar ya inmersos en ella. Sin embargo, los nuevos modelos apoyan la teoría de que no se trata de un fenómeno unidireccional sino que tanto la sociedad de origen como la receptora son dos dimensiones independientes que influyen una en la otra. Uno de los modelos de aculturación más conocido es el de Berry $(1989,2009)$. Este modelo de aculturación es bidimensional, y tiene el objetivo de conseguir entender el proceso por el que las personas migrantes se adaptan o no al lugar de destino. En concreto, el modelo de Berry consiste en responder a dos dimensiones: por una parte, si la persona migrante mantiene la identidad cultural de su grupo, y por otra parte si tiene contacto y adopta la identidad cultural del grupo de acogida (Berry, 2005; Sarriera, 2003). Según las respuestas a ambas preguntas, sean positivas o negativas, se pueden obtener cuatro dimensiones sobre la actitud de las personas migrantes ante el proceso de aculturación: "integrativa", la cual se da cuando la persona migrante continua con sus valores culturales de origen y a su vez mantiene relaciones con personas de culturas diferentes; "separación" que sucede cuando continua con su cultura pero no mantiene relaciones con otros grupos culturales; "asimilación" cuando la cultura propia del migrante no tiene continuidad y se relaciona con grupos de otras culturas, y por último la "marginación/exclusión" que se da cuando no se tiene el sentimiento de pertenecer a ninguna de las culturas y se evita la relación con ambos grupos (origen y acogida).

Según Salvador, Pozo, y Alonso (2010), "un individuo o un grupo pueden adoptar cuatro estrategias de aculturación, en función de su orientación en dos dimensiones independientes: el mantenimiento de la propia cultura y el contacto con la sociedad receptora" (p. 56).

Por otro lado, otros modelos como el Modelo Aplicado de Aculturación Relativa (MAAR) sostiene que la:

“Diferencia entre el plano real (estrategias) y el plano ideal (actitudes) del proceso de aculturación, es decir, entre las opciones puestas en práctica por los inmigrantes (o percibidas por los autóctonos) en su nueva sociedad de acogida (estrategias de aculturación), y las opciones que preferirían ambas poblaciones, en caso de poder elegir (actitudes de aculturación)" (Rojas, Sayans \& Navas, 2012, p. 71).

Por tanto, el modelo MAAR considera que los individuos (tanto del país de origen como del grupo inmigrante) utilizan estrategias y actitudes de aculturación diferentes dependiendo del ámbito 
sociocultural: público (político, bienestar social, laboral) o privado (ámbito familiar, religioso, principios), y es el país de destino, al que acude el inmigrante, el que establece el proceso de aculturación.

Por ello, ser competente en 'interculturalidad' se ha convertido en una pieza clave para todas las personas que están en contacto con otras de diferentes culturas, es decir, una competencia que favorece la convivencia y la ciudadanía en contextos multiculturales e interculturales (Carmona, Van der Zee y Van Oudenhoven, 2013).

\subsection{Competencia intercultural}

La competencia intercultural se define como aquel comportamiento que es efectivo y apropiado en un contexto de comunicación y socialización intercultural (Deardorff, 2011).

En esta competencia es inevitable que no se tengan en cuenta otros procesos cognitivos yactitudinales, como por ejemplo la educación de la persona, las experiencias previas, habilidades o aptitudes, entre otras muchas. Pero adquirir la capacidad de adecuación de un comportamiento a una situación intercultural debe ser el objetivo de la educación en esta materia.

En la Figura 1 se resume, según Deardorff (2011), cuáles son las herramientas fundamentales para ser competente interculturalmente. Pero es importante recalcar que en la mayoría de los casos no se alcanza un punto máximo o un aprendizaje completo en esta ni en otras muchas competencias, sino que es un aprendizaje continuo y voluble y que dependiendo del sujeto, contexto y experiencia se tendrá un comportamiento u otro.

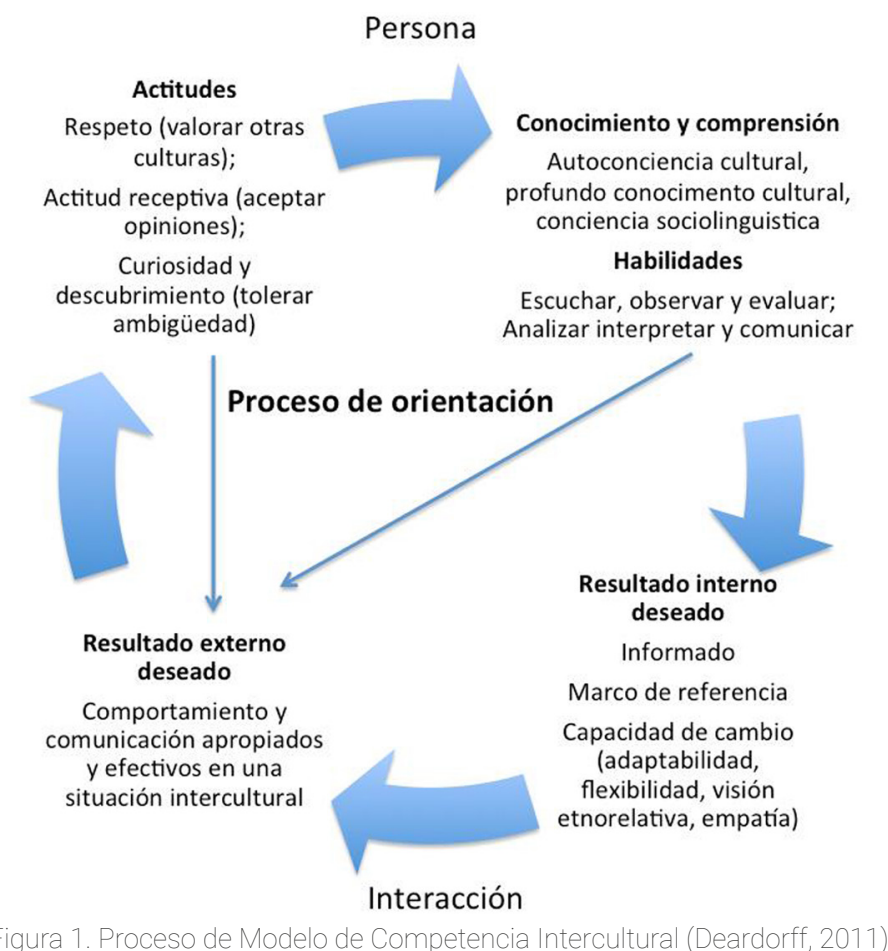


El modelo de la Figura 1 recoge los componentes que deben cumplirse para que una persona tienda a la interculturalidad, sin embargo, existen otras competencias que están estrechamente relacionadas con el aprendizaje intercultural y poseerlas favorece que los espacios que actualmente son multiculturales se encaminen hacia nuevos contextos interculturales.

Lo esencial de analizar cómo definir una persona intercultural radica en averiguar si la interculturalidad se educa, se aprende, cómo se transfiere y qué esferas facilitan espacios interculturales.

En resumen, se habla de la importancia que supone la llegada de la diversidad cultural y cómo se afronta en las vidas de los estudiantes. Es una realidad a la que debe hacer frente formando al profesorado primero y, segundo, al alumnado, de la mejor manera posible en competencias para la convivencia y la interculturalidad (Cruz Rodríguez, 2015). Programas, actividades e iniciativas que faciliten la adaptación de los estudiantes a contextos donde conviven diferentes culturas deben realizarse en todo tipo de centros educativos y a cualquier nivel académico (Ballesteros, Aguado y Malik, 2014; Latorre-Medina, Mateos-Claros, Olmedo-Ruiz y Esteban Ibáñez, 2017; Rodríguez Izquierdo, 2015; Vilà, 2006).

\subsection{Competencias relacionadas con el aprendizaje intercultural}

Existen diferentes competencias que ayudan a tener una adaptación a un contexto nuevo más favorable. En concreto, a partir del Modelo de Personalidad Multicultural-MPQ (Van der Zee y Van Oudenhoven, 2000, 2002), se ha comprobado una relación positiva entre los modelos de aculturación de Berry (2009) y las cinco competencias que componen el modelo. En concreto, la falta de desarrollo de las competencias de Iniciativa Social, Flexibilidad, Estabilidad Emocional, Apertura Mental, y Empatía Cultural dificultan las relaciones interpersonales y aumenta los niveles de ansiedad cuando se produce el encuentro entre dos culturas. A continuación, se reflexionará sobre la importancia y definición de cada competencia y de los múltiples beneficios para afrontar el nuevo contexto cultural.

Iniciativa social

La iniciativa social hace referencia a participar de manera activa en situaciones y contextos sociales demostrando interés, iniciativa y disposición en las distintas actividades e interacciones sociales o colectivas que se produzcan (Van der Zee y Van Oudenhoven, 2013).

Existen diferentes prácticas y competencias que se relacionan con la iniciativa social. Por ejemplo, ser capaz de reconocer una necesidad donde actuar, organización y habilidad para trabajar en función de unos objetivos, autoconocimiento y autoestima para implicarse de manera crítica en aquellas acciones sociales en las que se quiera participar, entre otras muchas. 
Además, querer participar de un modo activo en las situaciones cotidianas facilita el acercamiento entre las diversas culturas. Es decir, si se colabora con las actividades diarias que se suceden en una sociedad multicultural, inevitablemente existirá un acercamiento entre culturas.

\section{Flexibilidad}

Consiste en la capacidad de interpretar las situaciones nuevas como un desafío positivo y adaptarse a esos nuevos contextos de acuerdo con la demanda que estos exijan (Van der Zee y Van Oudenhoven, 2013). Ser flexible conlleva poseer una elasticidad y una inteligencia que permiten comprender con cierta rapidez las necesidades de la situación cultural que se presenta. Asumir como válidos nuevos procesos e incorporarlos para situaciones similares futuras es el objetivo de ser flexible en contextos interculturales.

\section{Estabilidad emocional}

Duradero, fijo o constante son sinónimos de estabilidad. Sin embargo, en el contexto intercultural no se hace referencia al término estabilidad como algo fijo sino todo lo contrario: moldeable. Poseer la competencia de estabilidad emocional consiste en ser capaz de mantener o adaptarse a las situaciones culturales nuevas sin sufrir demasiado estrés emocional (Van der Zee y Van Oudenhoven, 2013). Durante los cambios es normal afrontar situaciones estresantes, no obstante, se puede ser más o menos hábil. En un choque cultural o en un proceso de aculturación, en ambas o una de las dos culturas, el sujeto puede sentir estrés debido a la pérdida de identidad cultural. Si ambas personas poseen capacidades interculturales, como por ejemplo la estabilidad emocional, esta les permitirá no sufrir una situación de estrés y ser conscientes de que está produciéndose un aprendizaje de ambas culturas. Estar calmado no quiere decir estar apático, sino mostrar una actitud positiva, es decir, ajustar las emociones a la situación.

\section{Apertura mental}

La capacidad propia del individuo de saber por sí mismo que su pensamiento o idea puede ser errónea y que eso le invite a escuchar los pensamientos de los otros sin prejuzgarlos de antemano suponiendo que su opinión es cierta. Y si trasladamos esto a la interculturalidad, se asumirá que otras actividades, normas o costumbres son igual de válidas que las propias pese a no ser compartidas. Ser abierto mentalmente consiste en "valorar las nuevas experiencias y el conocimiento de nueva gente, estando abiertos a nuevas culturas, nuevas costumbres, incluso nuevas y diferentes formas de actuar" (García, 2013, p.182). Así pues, esta es la única manera de apreciar y aprender de otras culturas fuera de nuestra zona de confort. 
Formarse una opinión preconcebida, normalmente negativa, de una cultura supone una actitud perjudicial para que la transferencia cultural sea bidireccional, para que se produzca lo que llamamos integración dentro del proceso de aculturación.

\section{Empatía cultural}

La empatía es una competencia que facilita el entendimiento entre las personas. Conocer cómo se siente una persona con la que has de comunicarte o compartir experiencias ayuda a la hora de enfatizar el proceso comunicativo y conseguir los objetivos de una conversación (Van der Zee y Van Oudenhoven, 2000, 2002, 2013). Sin embargo, la empatía cultural ayuda, no solo en el proceso comunicativo entre personas de diferentes culturas, sino que refuerza y propicia un mejor entendimiento entre culturas. Gracias a esta competencia se comprenden los sentimientos, pensamientos y comportamientos que van ligados a una cultura.

\section{Competencia comunicativa}

La competencia comunicativa consiste en saber actuar con los interlocutores de la mejor manera posible para que se logre el objetivo final: transmitir una información. En concreto, según la Orden ECD/65/2015, de 21 de enero, por la que se describen las relaciones entre las competencias, los contenidos y los criterios de evaluación de la educación primaria, la educación secundaria obligatoria y el bachillerato:

"La competencia en comunicación lingüística (CCL) puede ser definida como el resultado de la acción comunicativa dentro de prácticas sociales determinadas, en las cuales el individuo actúa con otros interlocutores yatravés de textos en múltiples modalidades, formatosy soportes" (Chen, Benet-Martínez y Bond, 2008). En la competencia en comunicación lingüística se puede destacar la interacción de los siguientes componentes: lingüístico, pragmático-discursivo, socio-cultural, estratégico, y personal. De estos componentes, es necesario destacar, por un lado, el componente lingüístico relacionado con el aspecto sociocultural, el cual incluye dos dimensiones que están referidas al conocimiento del mundo y la dimensión intercultural, y por otro lado, el componente estratégico que permite al individuo superar las dificultades y resolver los problemas que surgen en el acto comunicativo. Asimismo, también forman parte de este componente las estrategias generales de carácter cognitivo, metacognitivo y socioafectivas que el individuo utiliza para comunicarse eficazmente, aspectos fundamentales en el aprendizaje de las lenguas extranjeras. Y, por último, el componente personal que interviene en la interacción comunicativa en tres dimensiones: la actitud, la motivación y los rasgos de personalidad. Este proceso contiene un componente que facilita la comunicación entre diferentes culturas. Gracias a la competencia comunicativa podemos conocer distintas culturas del mundo y desarrollar un componente socioafectivo en las relaciones interculturales (Comisión Europea, 2014; Gil Serra y Roca-Piera, 2011). 


\subsection{El programa Erasmus para estudiantes}

El Programa Erasmus es un programa que ha facilitado que más de 3 millones de estudiantes se formen en otro país y contribuyan así a la movilidad entre instituciones de educación superior. Estos programas de movilidad actualmente se han convertido en una línea fundamental de actuación, que promueve el intercambio entre universidades europeas en materias de contenidos, metodologías, recursos y, por supuesto, en potenciar y transmitir conocimiento (Belvis, Pineda y Moreno, 2008).

El programa Erasmus no solo consiste en la movilidad entre estudiantes, profesorado y personal de universidades europeas, sino que también facilita la cooperación entre instituciones en beneficio del aprendizaje cultural, lingüístico y académico.

En cuanto a los beneficios, es necesario destacar que el más importante que se obtiene gracias a este programa es ampliar las posibilidades de inserción laboral, así como fomentar competencias como la tolerancia, la confianza en uno mismo o la capacidad de reconocer virtudes y defectos en uno mismo (Comisión Europea, 2014). En este estudio de la comisión, otros datos significativos son que aquellas personas que se han ido de Erasmus tienen la mitad de probabilidades de sufrir desempleo, un 64\% de los empresarios encuestados acostumbra a dar más responsabilidad a los titulados con experiencia en otros países o, también, es mayor la probabilidad de estar en un puesto de dirección si se ha cursado el Erasmus.

\subsection{Las competencias interculturales y el programa Erasmus}

Un análisis fundamental que relaciona al estudiante Erasmus con las competencias interculturales se encuentra en el estudio de Alfranseder (2012) citado por Murillo (2014). En este estudio, distintas habilidades que se mejoran a través del programa Erasmus, menciona que las más destacadas son los conocimientos de idiomas y la capacidad de trabajar en entornos interculturales, pero va más allá, en el ámbito de la interculturalidad no solo se mejora la empleabilidad o acciones laborales, sino que se adquieren conocimientos sobre otras culturas, se mejora la competencia comunicativa, es decir la habilidad de comunicarse con personas de otros países o también se evoluciona hacia una "mente más abierta".

Por otro lado, la Comisión Europea (2014) examina en un estudio en diferentes países de la UE un aspecto muy relevante para el Espacio Europeo de Educación Superior (EEES): que el estudiante sea consciente de su propio aprendizaje. Uno de los resultados del estudio es que más del $90 \%$ de aquellos que participaron en el programa de movilidad Erasmus fueron conscientes de que habían mejorado en sus habilidades sociales, en el conocimiento de otros países, en la capacidad de trabajar e interactuar 
con personas de diferentes culturas, en su nivel de estabilidad emocional, en el conocimiento de idiomas y en la competencia comunicativa.

En síntesis, si durante el periodo en que el estudiante está en el extranjero está obligado a hacer frente a otras realidades diferentes a las de su cultura, y si el hecho de enfrentarse a otras realidades culturales - "el creciente contacto con miembros pertenecientes a otras culturas, no solo con los del país de recepción- promueve la sensibilidad cultural, la apertura mental y el cambio de perspectiva cognitiva hacia una actitud etnorrelativa" (Pozo y Aguaded, 2012, p. 456) es necesario continuar invirtiendo en programas de movilidad.

Por ello, ser competente en interculturalidad se ha transformado en una herramienta útil para todas las personas que están en contacto con otras personas de diferentes culturas, es decir, una competencia que favorece la convivencia y la ciudadanía en contextos multiculturales e interculturales (Carmona, et al, 2013; Van der Zee y Van Oudenhoven, 2002, 2013).

El objetivo del estudio es analizar de forma exploratoria las competencias interculturales del alumnado que solicita o no el programa Erasmus. Como se ha mencionado anteriormente, en estudios previos ya se ha analizado el perfil económico y cultural del estudiante que cursa el programa de movilidad, sin embargo, existen menos estudios sobre el perfil del alumnado que está pensando en realizar una estancia de movilidad durante sus estudios. Además, se examinarán qué beneficios el alumnado percibe que se obtiene tras participar en estos programas de movilidad, así como si se les informa y forma a los alumnos para que estén preparados para la movilidad.

\section{Método}

En el estudio se utilizó un diseño de carácter cuantitativo descriptivo y de tipo transversal con alumnado universitario.

\subsection{Participantes}

Mediante un muestreo no probabilístico intencional, la muestra se compuso por estudiantes españoles procedentes del área de ciencias sociales de la Universitat de València entre los cursos de grado de $1^{\mathrm{er}}$ a $3^{\mathrm{er}}$ año durante el $2016(n=59)$. Los participantes que han respondido a los cuestionarios están cursando las siguientes titulaciones: Pedagogía (6.8\%), Magisterio (69.5\%), Sociología (3.4\%) y Trabajo Social (20.3\%). La media de medad de los participantes es de 19.92 (un máximo de 27 y un mínimo de 18) con una desviación típica de 1.56. El número de mujeres que ha contestado al cuestionario ha sido de 49 (83.1\%) y un total de 10 hombres (16.9\%). Casi en la totalidad de encuestados, los estudios finalizados que poseían en el momento de realizar el cuestionario son Bachillerato 93.2\% y un 6.8\% 
un Ciclo de Grado Superior, ninguno de ellos realizó la prueba de acceso para mayores de 25 años. La nacionalidad de todos los encuestados es española.

\subsection{Instrumento}

Para realizar esta investigación se ha utilizado un cuestionario para medir las competencias interculturales, comunicativa, y variables descriptivas (mencionadas en el apartado anterior), así como el nivel académico de los progenitores de los encuestados, ya que puede ser una variable relevante en cuanto al nivel socioeconómico de las familias en las que el alumnado suele solicitar un programa Erasmus.

Las competencias interculturales se han medido a través de la forma corta del cuestionario (MPQ-SF, Van der Zee, Van Oudenhoven, Ponterotto y Fietze, 2013), adaptado para muestras españolas de la original de 91-ítems Multicultural Personality Questionnarie (MPQ; Van der Zee y Van Oudehonven, 2002). El cuestionario se compone de cinco dimensiones que miden: Empatía cultural, Estabilidad emocional, Flexibilidad, Iniciativa social, y Mente abierta. Los ítems se respondieron en una escala de respuesta tipo Likert en un rango de 1 (totalmente en desacuerdo) a 5 (totalmente de acuerdo).

Para medir la competencia comunicativa, se han diseñado seis ítems ad hoc que permiten medir esta competencia (p. ej., "me comporto igual conversando con una persona de mi misma cultura que con otra que no lo es"), en una escala de respuesta de 1 (totalmente en desacuerdo) a 5 (totalmente de acuerdo).

Para analizar la consistencia interna de cada una de las dimensiones se ha analizado el índice alfa de Cronbach para cada una de ellas y los resultados han sido los siguientes: empatía cultural a = .85; apertura mental $a=.87$; iniciativa social $a=.74$; flexibilidad $a=.86$; estabilidad emocional $a=.65 ; y$ competencia comunicativa $a=.72$. Se considera que valores superiores a .7 y .8 son suficientes para garantizar la fiabilidad de la prueba, pero debido a las características de la investigación, una muestra reducida, se ha admitido como válida la competencia de Estabilidad Emocional, la única inferior a .70.

\subsection{Procedimiento}

La selección de la muestra se realizó de manera no aleatoria y la participación de los encuestados fue voluntaria y totalmente anónima. El cuestionario fue administrado de manera on-line y la condición para participar en el estudio era estar cursando un grado del área de ciencias sociales en la UV en cualquiera de los cursos excepto cuarto. El cuestionario se administró a través de la herramienta de Google Doc. 


\subsection{Análisis de datos}

Los datos recogidos se han analizado con el programa estadístico SPSS 2.0 para Mac. El primer paso consistió en analizar todos los datos descriptivos de la muestra. El segundo paso fue analizar la normalidad; los resultados de la prueba indicaron normalidad para todas las variables objeto de estudio. En tercer lugar, se empleó la prueba t de Student para observar si existían diferencias entre los grupos generados a través de la variable "solicita o no el programa Erasmus".

\section{Resultados}

Los resultados descriptivos indican que tiene intención de cursar el programa Erasmus un 50.8\%, frente a un 49.2\% que no tiene intención de realizar ninguna movilidad durante la realización del Grado. Para aquellos que marcaron que sí desean realizar el programa Erasmus+, hemos extraído una serie de resultados interesantes. El estudio de preferencias de movilidad reveló que la gran mayoría del alumnado encuestado que realizaría este programa, sería en la modalidad de "Estudios" (70\%), seguido de la opción, "Aún no lo sé" (16.7\%), "Ambos" (10\%) y la modalidad "Prácticas" el porcentaje más bajo de todos (3.3\%).

Respecto a la preferencia de duración, un 60\% del alumnado que solicita el Erasmus estaría interesado en realizar una estancia menor, de tan solo un cuatrimestre, frente a un porcentaje más bajo que preferiría que la estancia fuese de todo el curso (36.7\%). Existe un tanto por cien de perdidos que no ha respondido a esta pregunta (3.3\%).

En cuanto a la formación de las familias de las personas encuestadas, es interesante resaltar que las madres y padres del alumnado que va a solicitar la beca Erasmus tienen un mayor nivel de formación (en particular en educación superior) que los que no tienen intención de solicitar la beca Erasmus. De aquellos que sí quieren estudiar parte de su Grado en otras universidades europeas, el idioma preferido por la inmensa mayoría es el inglés (66.7\%), seguido de por el italiano, con un respetable porcentaje (26.7\%). El porcentaje que desea cursar la beca en francés y portugués es el mismo y se caracteriza por ser muy inferior al resto (3.3\%). 


\begin{tabular}{|c|c|c|c|c|c|c|}
\hline & \multicolumn{2}{|c|}{$\begin{array}{l}\text { Desea solicitar } \\
\text { Beca movilidad }\end{array}$} & \multicolumn{2}{|c|}{$\begin{array}{l}\text { No desea solicitar } \\
\text { Beca movilidad }\end{array}$} & \multirow[t]{2}{*}{$t$} & \multirow{2}{*}{$\mathrm{p}$} \\
\hline & $M$ & DT & $M$ & DT & & \\
\hline Estabilidad Emocional & 3.2 & .69 & 3.07 & .70 & .63 & .53 \\
\hline Empatía Cultural & 4.09 & .44 & 3.94 & .66 & .97 & .33 \\
\hline Apertura Mental & 3.97 & .74 & 3.29 & .77 & 3.43 & $.001^{\star \star x}$ \\
\hline Iniciativa Social & 3.64 & .51 & 3.33 & .74 & 1.86 & .07 \\
\hline Flexibilidad & 2.95 & .71 & 2.61 & .92 & 1.60 & .12 \\
\hline Competencia Comunicativa & 4.19 & .46 & 3.86 & .56 & .10 & $.01^{\star \star}$ \\
\hline
\end{tabular}

Nivel de significación: * $p \leq .05,{ }^{* *} p \leq .01,{ }^{* \star *} p \leq .001$

\section{Tabla 1. Diferencia de medias en competencias interculturales entre los grupos}

Los resultados de la Tabla 1 indican en general que las medias en todas las dimensiones de competencias interculturales y comunicativa analizadas son más altas para el alumnado que pretende solicitar una beca Erasmus que los que no pretenden solicitarla.

En relación al análisis estadístico inferencial, como se observa en la Tabla 1, sí existen diferencias significativas en las dimensiones Apertura Mental y Competencia Comunicativa entre alumnado que tiene intención de solicitar la beca Erasmus y el alumnado que no tiene intención de solicitarla, más concretamente los alumnos que tenían la intención de solicitar obtuvieron mayores puntuaciones en el nivel de competencia lingüística y en apertura mental. Sin embargo, no se encontraron diferencias entre los alumnos con intención de pedir una Erasmus y los que no tienen la intención de pedir en estabilidad emocional, empatía cultural, iniciativa social y flexibilidad.

\section{Conclusiones}

La finalidad de esta investigación era averiguar si existían diferencias en el nivel de competencias interculturales entre los alumnos que desean realizar el programa de movilidad europeo Erasmus y aquellos que no tienen intención o no desean cursarlo.

Conocer el bagaje, la historia, la cultura de los estudiantes que solicitan programas de movilidad ha despertado interés en la comunidad científica y son numerosas las investigaciones que se preocupan en conocer qué lleva al estudiante a solicitar una beca de estas características (Bustillo Mesanza y Rodríguez González, 2011; Lesjak, Juvan, Ineson,Yap y Podovsovnik, 2015). En un estudio anterior, Belvis et al. (2007) Ilevaron a cabo una investigación que tenía como objetivo conocer cuáles eran los motivos de movilidad. Los resultados muestran cuatro factores determinantes a la hora de elegir cursar un periodo de la formación académica en el extranjero: influencias personales, motivos académicos-profesionales, motivos personales y otros motivos. Por ejemplo, se habla por supuesto 
del peso que tiene el nivel económico y cultural de los progenitores. "Cuanto más elevado es el estatus socioeconómico y cultural, se dan más posibilidades de participación en los programas" (Belvis, et al., 2007, p. 5). La movilidad no solo supone un trampolín académico y profesional para quien lo cursa, es también un motor de aprendizaje personal y cultural gracias a la inmersión en la cultura del país de destino.

Sin embargo, un dato interesante y que resulta relevante para esta investigación es que el segundo motivo escogido por los estudiantes en la variable de motivos personales es el "atractivo lúdicocultural del destino", es decir, estar interesado en la cultura del país donde tienen intención de realizar la movilidad.

En el estudio de Villarroel, Vizcarra, Camino, y Aristizabal (2011) se observaron diferencias entre un grupo que ya estaba inscrito como futuro estudiante Erasmus y otro grupo que no, indicando puntuaciones más altas en apertura mental, responsabilidad, diversidad cultural, eficacia e interconectividad en aquellos estudiantes que estaban inscritos en el programa Erasmus.

En referencia a las limitaciones del estudio, habría sido necesaria una muestra más amplia. Como se ha observado, no existen diferencias significativas en todas las dimensiones de competencias estudiadas, tan solo en dos, por tanto, el perfil intercultural del futuro estudiante Erasmus no es tan diferente del de un estudiante que no quiere cursar un programa de movilidad. Sin embargo, sí resultan más significativas, como se ha visto en otras investigaciones, las diferencias en el nivel socioeconómico de las familias. Es decir, el perfil del alumnado que vive la experiencia Erasmus es el de un alumno con un nivel socioeconómico por encima de la media del resto de compañeros.

La educación superior procura formar a los estudiantes en materias y competencias que les hagan crecer intelectualmente, socialmente y les faciliten la incorporación al mundo laboral. Sin embargo, también son necesarias otras competencias específicas que ayudan a la adaptación a un nuevo entorno cultural y de trabajo en otros países si ese es el futuro de muchos estudiantes (Wolff y Borzikowsky, 2018). La universidad brinda este aprendizaje con programas, actividades y acciones que favorecen el aprendizaje en competencias interculturales. Un ejemplo es el programa Erasmus.

El programa Erasmus es un promotor de diferentes competencias, entre ellas, la interculturalidad. Una estancia en el extranjero, como las realizadas en el programa Erasmus, invita al sujeto a estar en permanente contacto y negociación con otra cultura. Esto obliga a desarrollar sistemas y estrategias que permitan adaptarte al país de destino y asentar en los procesos habituales los cambios y un posterior ajuste sociocultural (Pozo y Aguaded, 2012).

Hoy en día, cada vez se concibe y entiende menos al individuo en solitario y en un único contexto social. Intercambios debidos a la globalización y a la movilidad, dentro y fuera de Europa, han 
provocado interconexiones que necesitan nuevas habilidades y competencias para que el desarrollo de las nuevas relaciones entre los individuos sea positivo. El Erasmus potencia habilidades para que las personas aprendan a desenvolverse e integrarse en diferentes entornos y sociedades. Al tratarse de un programa educativo, es importante, como decíamos, para la comunidad educativa mantenerlo y mejorarlo. Desde una perspectiva pedagógica, es fundamental educar en materia de competencias a favor de la movilidad, por lo tanto, acciones educativas como esta deben ser una prioridad, ya que constituyen aprendizaje muy útil para el desarrollo integral del individuo, para que sea crítico y consiga adaptarse a la sociedad con el mayor éxito posible.

\section{Referencias}

Alves, R. D. y de la Peña, A. (2013). Culture shock: adaptation strategies. Revista Nebrija de Lingüística Aplicada, 13.

Ballesteros, B., Aguado, T. y Malik, B. (2014). Escuelas para todos: diversidad y educación obligatoria. Revista Electrónica Interuniversitaria de Formación del Profesorado, 17(2), 93-107. doi: https://doi. org/10.6018/reifop.17.2.197351

Belvis, E., Pineda, P. y Moreno, M. V. (2007) La participación de los estudiantes universitarios en programas de movilidad: factores y motivos que la determinan. Revista Ibero-Americana de Educación, 42(5), 1-11.

Berry, J. W. (1990). Psychology of Acculturation. En: J. Berman (Ed.), Cross Cultural perspectivas: Nebraska Symposium of Motivation (457-488). Lincoln: University of Nebraska Press.

Berry, J. W. (2005). Acculturation: Living successfully in two cultures. International Journal of Intercultural Relations, 29(6), 697-712. doi:https://doi.org/10.1016/j.ijintrel.2005.07.013

Berry, J. (2008). Globalisation and acculturarion. International Journal of Intercultural Relations, 32, 328-336. doi:https://doi.org/10.1016/j.ijintrel.2008.04.001

Berry, J. (2009). A critique of critical acculturarion. International Journal of Intercultural Relations, 33, 361-371. doi: https://doi.org/10.1016/j.ijintrel.2009.06.003

Berry, J., Kim, U., Power, S., Young, M. y Bujaki, M. (1989). Acculturation attitudes in plural societies. AppliedPsychology:An InternationalReview, 38,185-206. doi:https://doi.org/10.1111/j.1464-0597.1989. tb01208.x

Bustillo Mesanza, R. y Rodríguez González, C. (2011). The determinants of international student mobility flows: an empirical study on the Erasmus programme. Higher Education, 62, 413-430. https:// doi.org/10.1007/s10734-010-9396-5 
Carmona, C., Van der Zee, K. y Van Oudenhoven J. (2013). Competencias interculturales: un aspecto clave para la internacionalización. En Gacel-Ávila, J. y Orellana N. (Ed.), Educación superior, gestión, innovación e internacionalización, (pp. 195-214). Valencia: Universitat de València.

Chen, S. X., Benet-Martínez, V.y Harris Bond, M. (2008). Bicultural Identity, bilingualism, and psychological adjustment in multicultural societies: immigration-based and globalization-based acculturation. Journal of personality, 76(4), 803-838. doi: https://doi.org/10.1111/j.1467-6494.2008.00505.x

Comisión Europea. (2014). The Erasmus impact study. Luxembourg: Publications Office of the European Union.

Cruz Rodríguez, E. (2015). La interculturalidad en las políticas de educación intercultural. Praxis \& Saber, 6(12), 191-207. doi:https://doi.org/10.19053/22160159.3769

Deardorff, D. (2011). Assessing Intercultural Competence. New Directions for Institutional Research, 149, 65-77. doi:https://doi.org/10.1002/ir.381

Escámez, J. (1994). La tolerancia entre culturas como Procedimiento para la paz. Teoría de la Educación, 6, 23-3.

García Prieto, E. (2013). ¿Qué es el programa Erasmus? Movilidad internacional de estudiantes y docentes: 25 años de éxito. Madrid: Pirámide.

Giddens, A. (1991). Sociología. Madrid: Alianza.

Gil Serra, A. F. y Roca-Piera, J. (2011). Movilidad virtual, reto del aprendizaje de la educación superior en la Europa 2020. RED. Revista de Educación a Distancia, 26, 1-16. Recuperado de http://www.um.es/ $\mathrm{ead} / \mathrm{red} / 26 /$

Latorre-Medina, M. J., Mateos-Claros, F., Olmedo-Ruiz, F. J. y Esteban Ibáñez, M. (2017). Modelos culturales en un contexto multicultural. IJERI: International Journal of Educational Research and Innovation, 9, 31-45.

Lesjak, M., Juvan, E., Ineson, E. M., Yap, M. H. T., y Podovsovnik, E. (2015). Erasmus student motivation: Why and where to go? Higher Education, 70, 845-865. doi:https://doi.org/10.1007/s10734-015-9871-0 Murillo, J. (2014). El programa Erasmus y el desarrollo de capacidades. Un análisis multidimensional. Tesis Doctoral. Universitat de València, Valencia.

Pozo, C. y Aguaded, J. I. (2012). El programa de movilidad ERASMUS: motor de la adquisición de competencias interculturales. Revista de Investigación Educativa, 30(2), 441-458. 
Rodríguez Izquierdo, R. M. (2015). Competencias genéricas en la enseñanza superior a través de los programa de internacionalización. Revista Complutense de Educación, 26(1), 81-100. doi:https://doi. org/10.5209/rev_RCED.2015.v26.n1.42598

Rojas, A. J., Sayans-Jiménez, P. y Navas, M. (2012). Similitud percibida y actitudes de aculturación en autóctonos e inmigrantes. International Journal of Psychological Research, 5(1), 70-78.

Sáenz, M. C. L. (2015). Diálogo intercultural ¿Una utopía del siglo XXI? Pensamiento. Revista de Investigación e Información Filosófica, 71(265), 73-94. doi:https://doi.org/10.14422/pen.v71.i265. y2015.004

Sarriera, J. C. (2003). Estudios actuales sobre la aculturación en latinos: revisión y nuevas perspectivas. Revista Interamericana de Psicología, 37(2), 341-364.

Van der Zee, K. y Van Oudenhoven, J. P. (2000). The Multicultural Personality Questionnaire: A multidimensional instrument of multicultural effectiveness. European Journal of Personality, 14(4), 291-309.

Van der Zee, K. y Van Oudenhoven, J. P. (2002). Multicultural Personality Questionnarie. International Journal of Intercultural Relations, 26, 679-694. doi:https://doi.org/10.1002/10990984(200007/08)14:4<291:.AID-PER377>3.0.C0;2-6

Van der Zee, K. y Van Oudenhoven, J. P. (2013). Culture Shock or Challenge? The Role of Personality as a Determinant of Intercultural Competence. Journal of Cross-Cultural Psychology, 44, 928-940. doi:https://doi.org/10.1177/0022022113493138

Van der Zee, K., Van Oudenhoven, J. P., Ponterotto, J. y Fietzer, A. (2013). Multicultural Personality Questionnaire: Development of a Short Form. Journal of Personality Assessment, 95(1), 118-124. doi:https://doi.org/10.1080/00223891.2012.718302

Vilà, R. (2006). La dimensión afectiva de la competencia comunicativa intercultural en la Educación Secundaria Obligatoria: Escala de Sensibilidad Intercultural. Revista de investigación educativa, 24(2), 353-372.

Villarroel, J. D., Vizcarraa, M. T., Camino, I. y Aristizabal, P. (2011). International Mobility and Psychological Factors: A Related Study for Teacher Training. Procedia, Social and Behavioral Sciences, 30, 2124-2128. doi:https://doi.org/10.1016/j.sbspro.2011.10.412

Ward, C. y Kennedy, A. (1994). Acculturation strategies, psychological adjustment, and sociocultural competence during cross-cultural transitions. International Journal of Intercultural Relations, 18(3), 329-343. doi:https://doi.org/10.1016/0147-1767(94)90036-1 
Wolff, F. y Borzikowsky, C. (2018). Intercultural Competence by International Experiences? An Investigation of the Impact of Educational Stays Abroad on Intercultural Competence and Its Facets. Journal of Cross-Cultural Psychology. doi:https://doi.org/10.1177/0022022118754721 